Revue d'histoire de l'Amérique française

ZWB REVUE D.HISTOIRE DE L'AMÉRIQUE FRANÇAISE

\title{
Une émeute à Lachine contre la " conscription » (1812)
}

\section{Jean-Pierre Wallot}

Volume 18, numéro 1, juin 1964

URI : https://id.erudit.org/iderudit/302344ar

DOI : https://doi.org/10.7202/302344ar

Aller au sommaire du numéro

Éditeur(s)

Institut d'histoire de l'Amérique française

ISSN

0035-2357 (imprimé)

1492-1383 (numérique)

Découvrir la revue

Citer cet article

Wallot, J.-P. (1964). Une émeute à Lachine contre la « conscription » (1812).

Revue d'histoire de l'Amérique française, 18(1), 112-137.

https://doi.org/10.7202/302344ar d'utilisation que vous pouvez consulter en ligne.

https://apropos.erudit.org/fr/usagers/politique-dutilisation/ 


\section{UNE ÉMEUTE À LACHINE CONTRE LA "CONSCRIPTION" (1812)}

Ulcérés par des années d'impuissance, d'humiliations et de restrictions de toutes sortes, les États-Unis, en juin 1812, rompirent une paix déjà fragile. Le 4 , la Chambre des représentants, divisée, optait pour la guerre 79 voix contre 49. La majorité au Sénat, également déchiré, se rallia à la mesure 19 voix contre 13. Le même jour, 18 juin, James Madison apposait sa signature à l'acte de guerre. ${ }^{1}$ Depuis trop longtemps, à ses yeux, l'orgueilleuse Angleterre écumait le commerce et violait la neutralité de son pays. Elle faisait arraisonner les navires américains en haute mer: insulte flagrante au pavillon national, ses officiers y triaient et conscrivaient arbitrairement de prétendus sujets britanniques, souvent en fait des citoyens américains. Enfin, les Britanniques ne tisonnaient-ils pas la haine féroce des sauvages à l'égard des États-Unis, cause du harcèlement des colons au nord-ouest ? ${ }^{2}$

La France aussi piétinait les "droits" des neutres. Mais petitement, à la mesure de sa puissance maritime: ses quelques navires encore à flot étaient claquemurés dans les ports français par la flotte britannique, toute-puissante sur mer et à l'affût d'une proie aussi vulnérable. A cause de cette suprématie, c'était surtout la marine anglaise qui torpillait le commerce maritime des neutres. C'était donc la nation anglaise qui allait écoper de la fureur d'une partie de la nation américaine - l'autre, la classe des armateurs, préférant une riche servilité envers la "libre" Angleterre à une neutralité trop austère ou la servitude ruineuse

${ }^{1}$ La Gazette de Québec, 29 juin 1807; Richard Hofstadter, Great Issues in American History (2 vols., New York, 1958), 1: 234; Thomas A. Bailey, A Diplomatic History of the American People (New York, 1947), 136-140.

2 Voir le message de guerre du président Madison au Congrès, le 1er juin 1812, dans Richard Hofstadter, Great Issues [...], 1: 234-237. 
sous le "tyran" d'Europe. Colère qui pourtant ne pouvait éclabousser les îles britanniques, lointaines et inexpugnables. Elle devait donc s'assouvir sur le British North America, parce que faible et accessible. On canarderait facilement, s'illusionnait-on, cette cible de choix, victoire éclatante propre à ressusciter la fierté nationale avilie. Cette perspective agréable - une bonne morsure dans le flanc de la "fière Albion" - se doublait comme par hasard d'avantages non négligeables: soumission ou écrasement des sauvages; expulsion des Britanniques et de leur menace au nord; accaparement d'un pays vaste et riche par son réseau formidable de communications fluviales, un commerce payant, des terres fertiles, etc.

La déclaration de la guerre prit au dépourvu le cabinet anglais, bousculé par les événements. Aussi tard que le 4 juillet, il croyait possible d'éluder la catastrophe. ${ }^{3}$ Ce "wishful thinking" de Londres, heureusement, n'assoupissait pas le gouverneur Prévost dans la colonie. Et durant la session d'hiver, malgré les relents de cette rancune tenace qu'entretenait la Chambre d'assemblée à l'égard de Craig, ${ }^{4}$ le gouverneur réussit à manœuvrer celle-ci tant bien que mal. La Législature, en effet, améliora substantiellement l'acte de milice. La nouvelle loi habilitait le commandant-en-chef, en temps de paix, à décréter le tirage au sort de 2,000 miliciens parmi les célibataires de 18 à 30 ans, afin de les dresser durant une période annuelle ne dépassant pas trois mois. En cas de besoin, il pouvait verser ces miliciens dans le service actif pour deux années chacun au maximum. Enfin, la Législature affecta des sommes de $£ 12,000$ à la milice, de $£ 20,000$ à la sécurité de la province et de 330,000 à sa défense en cas d'invasion ou de guerre. ${ }^{5}$ A ce moment, Prévost n'entendait recourir à l'acte de milice qu'avec beaucoup de circonspection: sa méfiance s'alimentait aux nombreuses dénonciations que

3 Bathurst à Prévost (no 2), Downing Street [à l'avenir: D.S.], 4 juillet 1812, Archives publiques du Canada [AC], dépêches du Colonial Office [R.G. 7, G. 1], 5: 138.

4 Journal de la Chambre d'Assemblée du Bas-Canada [à l'avenir: $J C A B C]$ (1812), 39-59 et passim.

5 JCABC (1812), 59-63, 221-229, 255-263, 275-293, 333-335, etc. Prévost à Liverpool (no 40), Québec, 20 avril 1812, AC, Papiers du Colonial Office [M.G. 11], Q. 117, 2: 214 et suivantes. 
distillaient les rapports de son prédécesseur - Craig - "of the supineness of the Canadians, \& of the industry with which the seeds of disaffection \& disorder have for many years been sown amongst them ...". ${ }^{6}$ Toutefois, un mois plus tard, l'imminence de la guerre accula le gouverneur à l'action. Dans une lettre circulaire du 20 mai, il commanda, en vertu de l'acte de milice, la levée à travers la province de 2,000 jeunes hommes pour la première semaine de juin. ${ }^{7}$ Les États-Unis ne déclencheraient les hostilités qu'un mois plus tard.

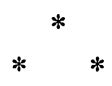

En général, les 2,000 miliciens se métamorphosèrent en soldats sans résistance. Sans doute, il y eut des réfractaires ici et là. Ils ne glissèrent pas sur cette pente nécessairement par "désaffection" à l'égard du gouvernement. Mais partout et toujours, en guerre et surtout en paix, les hommes n'aiment pas se faire enrégimenter. Et de fortes têtes, des individualistes irréductibles, des paysans enracinés dans leurs travaux, etc. cèdent à la tentation instinctive d'éprouver la force de l'autorité. Si celle-ci hésite, vacille, on se cambre et s'insurge avec succès contre ses ordres. Si au contraire, elle dompte les récalcitrants avec vigueur, leur résolution s'effrite rapidement.

Or en juin 1812, des circonstances nettement défavorables à l'autorité se conjuguaient à cette motivation normale et universelle. Il s'agissait fondamentalement d'une querelle entre Anglais. Depuis la conquête, on n'avait pratiquement jamais discipliné ni même réuni la milice d'ailleurs désarmée. Une première tentative en ce sens, en 1794, avait buté contre une obstruction généralisée et opiniâtre. Cependant, en 1801, alarmé par des rumeurs fantastiques au sujet d'une invasion probable du Canada par un corps expéditionnaire du Vermont, Milnes n'eut aucune

${ }^{6}$ Prévost à Liverpool (no 40), ibid., 218.

7 Lettre circulaire de l'adjudant-général Vassal de Monviel aux officiers de milice, Québec, 20 mai 1812, AC, Collection Baby [M.G. 22/6], 36: 101. Christie soutient que cette levée se fit en vertu d'un ordre général du 28 mai: voir Robert Christie, History of Lower Canada [...] (6 vols., Québec, 1848-1855), 2: 10. 
difficulté à placer sur un pied d'alerte la milice de la région montréalaise. Celle-ci se groupa avec rapidité et dans les meilleures dispositions, à l'étonnement du gouverneur et de ses conseillers. En 1807, à la suite de la raclée infligée par la frégate britannique Leopard à la Chesapeake, des États-Unis, le président Dunn fit alerter plusieurs milliers de miliciens. Galvanisée par son vieux "père", la population étala un zèle débordant et jamais égalé: en plusieurs endroits, le nombre de volontaires surpassa les besoins. Mais peut-être qu'à force de brandir le spectre américain, de crier au loup, cette évocation perdait-elle de son impact. On émoussait cet empressement en le faisant "répéter". En cette saison, les cultivateurs devaient trimer dur sur leurs terres. Depuis 1774 , des émissaires étrangers, américains et français, visitaient régulièrement la province et particulièrement les campagnes de la région de Montréal, leur terre de prédilection: ils inoculaient habilement leur venin dans une population ignorante. Enfin, ne sortait-on pas du "règne de la terreur" ?

Il y a plus. L'improvisation préside à ce premier rassemblement général de la milice. Ce qui décuple les difficultés déjà considérables de l'opération, tant pour les miliciens que pour le gouvernement. Ainsi, après avoir cheminé jusqu'à Pointe-auxTrembles, le campement général pour la région de Québec, les cent-soixante miliciens de Duchesnay sentent leur enthousiasme refroidir: ils n'ont ni paillasses ni lits pour se coucher, ni armes ni munitions pour pratiquer le tir. Même les plus zélés "montrent ouvertement leur mécontentement". ${ }^{8}$ Dans d'autres paroisses, l'insoumission des hommes est provoquée par la conduite des officiers, soit des vieillards de plus de soixante-dix ans, ignorants et impuissants, soit des tièdes ou des mous ou les deux à la fois. ${ }^{9}$

A Laprairie, point de ralliement dans le district de Montréal, on manque de sergents sachant le français pour "former les officiers et les sous-officiers et... accélérer les progrès de la

${ }^{8} \mathrm{~A}$. Juchereau Duchesnay à l'adj.-général de la milice (Vassal de Monviel), Pointe-aux-Trembles, 3 juin 1812, AC, Papiers de la milice du Bas-Canada [R.G. 9, IA 1], 3: dossier juin 1812 [s.p.].

${ }^{9}$ Lt-Colonel E. St-Dizier au même, Montréal, 20 juin, 1812, ibid. 
discipline...". ${ }^{10}$ De nombreuses lacunes indisposent les miliciens, "toutes... petites choses [qui] font murmurer des gens qui se trouvent là contre leur volonté" 11 - entendons: qui préfèreraient vaquer à leurs occupations, surtout en cette saison, au lieu de moisir dans l'inactivité et la pagaille. Dans VaudreuilSoulanges, à l'exception de quelques mutins, tous se comportent de manière louable: de Lotbinière conduit à Laprairie cinquanteet-un des cinquante-trois miliciens désignés par le sort. Mais avec quelle misère ! Certains, pour se nourrir, doivent emprunter de l'argent en route. De Lotbinière leur avance lui-même des fonds, finance leur transport de Montréal à Laprairie. ${ }^{12}$ Une mutinerie secoue le corps des "Voltigeurs canadiens", des réguliers coloniaux commandés par le major Charles de Salaberry le héros de Châteauguay: mauvaises conditions de vie (vivres insuffisants, logement inadéquat), renseignements inexacts répandus par un officier au moment de l'enrôlement. ${ }^{13}$ D'ailleurs, par suite de l'ignorance des miliciens, les gueulards peuvent manipuler leurs émotions assez facilement.

Presque partout, toutefois, on se débrouille bien. Les cent quatre miliciens de l'Ile Jésus exhibent beaucoup de gaieté et de loyauté, aux cris de "Vive le Roi". ${ }^{14}$ Malgré les retards, près de quatre cent dix hommes, apparemment "très contents", campent à Laprairie, le 10 juin. Après un compte rendu élogieux du

10 Hertel de Rouville au même, Laprairie, 10 juin 1812, ibid.

11 Le même au même, Laprairie, 18 juin 1812, ibid.

12 Chartier de Lotbinière au même, Montréal, 8 juin 1812, et le même au même, 13 juin, ibid.

13 Major Charles de Salaberry à N. Freer, secrétaire militaire, Fort Chambly, 18 juin 1812, AC, Papiers militaires britanniques, série "C" [R.G. 8, C], 696: 118-131. Prévost ne mâche pas ses mots: "I ascribe this unpleasant circumstance, in a great measure, to Captain Perrault's improper, impolitic and unauthorized advertisement in the Montreal Newspapers... I therefore desire you will remove from the minds of your men any remaining impressions left by it, which are contrary to your recruting instructions, and which are, in consequence, opposed to the spirit of the service the Voltigeurs are engaged to perform..." Et Prévost de pester contre la négligence coupable de certains officiers. Voir N. Freer au major Charles de Salaberry, Québec, 20 juin 1812, et Prévost au même, Québec, 21 juin 1812, ibid., 1218: 282-284.

14 J. Lacroix à V. de Monviel, Ile Jésus, 4 juin 1812, AC, R.G. 9, IA 1, 3: dossier juin 1812 [s.p.]. 
tirage au sort dans la région de Québec, la Gazette de Québec convient de ce que la "loi ... a quelques traits qui sont nouveaux et que plusieurs... regardent comme sévères", v.g. aucune exemption possible, même par substitution ou pour les apprentis, "l'objet de la loi étant d'exercer successivement toute la jeunesse (jeunes non mariés de 18 à 30 ans ...) du pays dans l'usage des armes". ${ }^{15}$ Malgré tout, les jeunes gens manifestent beaucoup de soumission et de zèle, une "disposition qui a toujours caractérisé le peuple de ce pays en pareilles occasions. Si l'on a entendu quelques murmures, ils n'ont été occasionnés que par la manière dont quelques compagnies ont fourni leur nombre de miliciens, et parce que dans une instance, tout le fardeau a tombé... sur une [même] classe de la société". ${ }^{16}$ Une semaine plus tard, le même journal exalte la conduite des recrues de Montréal: "nous ne nous rappelons pas avoir jamais vu un corps de plus beaux jeunes gens et qui donnassent plus de preuves de loyauté ... ${ }^{17}$ Même le Quebec Mercury, d'ordinaire grognon, module quelques compliments flatteurs à l'adresse des miliciens de la province, par suite de leurs efforts "honourable and spirited". Désormais rassuré, il peut se permettre de giffler verbalement les envahisseurs éventuels, car "they will find that Canadians have not degenerated... If English and French be considered as natural enemies, Canadians and Yankees are not less so": ${ }^{18}$ constatation ambiguë lorsqu'on connaît la haine féroce que voue le Mercury à tout ce qui est Français. ${ }^{19}$

Ces quelques faits n'ont pas la prétention de buriner un tableau complet de toute la situation. Mais ils en cernent certains aspects, en évoquent l'atmosphère générale. Dans un tel cafouillis, surtout au début, dans une telle saison - la plupart des miliciens sont cultivateurs - , dans de telles conditions matérielles et psychologiques, les résistances qui affleurent ici et là n'ont

15 Hertel de Rouville au même, Laprairie, 10 juin 1812, ibid.

16 La Gazette de Québec, 4 juin 1812.

17 Ibid., 11 juin 1812.

18 Quebec Mercury, 30 juin 1812.

19 Voir, à titre d'exemples, les articles de ce journal cités par JeanPierre Wallot, "La Querelle des prisons (1805-1807)", $R H A F, 14$ (1960): 400-404, 559-575. 
pas de quoi faire sourciller. On peut même se surprendre de leur petit nombre et de leur peu d'importance.

L'émeute à Lachine les surplombe de beaucoup, par sa gravité. Avec une spontanéité apparente, elle dégénéra presque en insurrection armée. Cette mutation comme naturelle implique l'existence d'un climat déjà survolté dans cette région. Dans la division de Pointe-Claire (depuis Lachine jusqu'à l'extrémité ouest de l'île de Montréal), on avait choisi cinquante-neuf hommes. Vingt-huit seulement parurent à Laprairie. De ce nombre, quatre s'enfuirent presque aussitôt. Le 29 juin, le Colonel StDizier, commandant du bataillon, dépêcha le major Leprohon dans cette région. Celui-ci devait dénicher et mettre aux arrêts les hommes manquant à l'appel, dont un dénommé Léveillé, de Ste-Geneviève, sourd aux avis répétés du capitaine de milice Thibodeau. ${ }^{20}$

Le même jour, Leprohon se mit en route, escorté de deux capitaines et d'une trentaine de miliciens. Il râfla deux déserteurs. Le lendemain, 30 juin, vers 8 heures a.m., lui et une quinzaine d'hommes firent irruption chez Léveillé et le capturèrent. On le fit monter dans une charrette. Puis le groupe se transporta jusque chez le capitaine Binet, où il arriva vers 9 heures a.m. Un comité de réception l'attendait: une bande d'hommes remuait déjà sur les lieux. Ils "allaient et venaient vers une grange et... refusaient de... dire ce qu'ils faisaient là". Leprohon s'enquit auprès de Binet s'ils allaient l'assister dans la chasse aux déserteurs. "Oui", mentit Binet. Affirmation contredite presque aussitôt par leur conduite. Ils se mirent à

20 Rapport du conseil exécutif (comité de Montréal) sur cette émeute [Rapport du comité de Montréal], 1er juillet 1812, AC, Procès-verbaux du conseil exécutif, livre d'état $\mathrm{G}[P V C E, G]: 253$. Aussi Témoignages des Colonels Deschambault et St-Dizier, et du major Leprohon dans le procès faisant suite à cette émeute, 19 août 1812 , et témoignage du Colonel St-Dizier, 20 août 1812, AC, M.G. 22/6, 32: 262-264, 276. A l'avenir: Témoignages de [nom (s)], [date(s)]: [page (s) ]. La Collection Baby comprend deux transcriptions complètes de ces procès: au volume 32 (ci-haut) et également au volume $73: 117-186$. 
ronchonner. Aucune loi, ergotaient-ils, n'avait été sanctionnée pour embrigader des miliciens. S'ils consentaient au recrutement de jeunes volontaires, "ils ne souffriraient pas qu'on les y forçât”. Un Anglais, Fraser, prévint alors Leprohon que la grange abritait d'autres hommes ainsi que des armes.

Préférant la discrétion à un hérö̈sme dangereux et inutile, Leprohon monta en voiture et voulut décamper. Mais sur la route, "un grand nombre d'hommes... bordaient le chemin et le suivaient de près et un grand nombre cherchaient à le devancer et à la fin, ils lui ont barré le chemin au nombre de 30 ou 40 qui se sont augmentés à plus de 100 et dont il a reconnu plusieurs qu'il venait de laisser chez le Capitaine Binet - près de 40 étaient armés".21 Avant son départ de chez Binet, en effet, Louis Paiement et Jacques Trottier avaient incité les autres à intercepter Leprohon, "aussitôt [qu'il] sera parti". ${ }^{22}$ Ceinturé rapidement par cette barrière houleuse, l'officier dut stopper. D'autant plus que certains braquaient leur fusil sur lui, exigeant la reddition du prisonnier. "Rendez-nous le prisonnier, marchanda Trottier, on ne vous fera point de mal." Vraisemblablement terrifié, Leprohon crut plus "prudent" de capituler: "ils paraissaient tous animés et violents."

Grisés de ce succès facile, les émeutiers délivrent le prisonnier en clamant: "Vive le Roi" ! Puis, plusieurs décident de filer Leprohon afin de faire élargir les deux autres prisonniers qu'ils croient chez le capitaine Ducharme. Ils font une halte chez le capitaine Dumouchel. Trottier, un de ceux qui ont cuisiné le coup, estomaque Leprohon lorsqu'à une question de celui-ci, à savoir s'ils veulent "faire une insurrection", il rétorque: "nous voulons faire une guerre civile" ! ${ }^{23}$ L'appétit vient en mangeant: un peu plus loin, chez Deslauriers, alors que Leprohon transfère de voiture, six ou sept miliciens le somment de restituer les deux prisonniers: "si on ne nous renvoie pas nos prisonniers, nous irons les chercher à Lachine." 24 "Nous avons-celui-là, se targuent

21 Témoignage du major Leprohon, 19 août: 264-265.

22 Témoignage de Louis-Hyacinthe Proulx, 19 août: 268-269.

23 Témoignage du major Leprohon, 19 août: 265-266.

24 Témoignage du milicien Robert Grant, 19 août: 266-267. 
Louis Paiement et Noël Legault, et il faut avoir ceux que $\mathrm{Mr}$ Leprohon a pris hier au soir." Une cinquantaine d'hommes talonnent Leprohon à nouveau, cette fois jusqu'à Lachine. "Nous voulons ravoir nos prisonniers, scandent-ils, nous reviendrons demain pour voir si vous avez ramené nos prisonniers." "Vous voulez donc faire une guerre civile?" - "Ma foi oui", fuse de nouveau cette réponse. ${ }^{25}$

C'est surtout cet affront, cette forfanterie qui hérissa les conseillers exécutifs de Montréal. D'après les premiers rapports, ils supputèrent le nombre des frondeurs à environ deux cents, dont plusieurs en armes.

Not contended with this insult offered to His Majesty's Government, the mob threatened that if the two men who had been sent prisoners to town were not given up by 12 o'clock today, they would come and take them out by force, and further threatened that they would go to Laprairie and bring away their men who were at Laprairie - ... information... was received this forenoon from Ste Genevieve behind Pointe Claire giving notice that the Habitants to the number of five hundred assembled and threatened to march early in the morning for the same evil purposes - One of the unlawfull assembly declared that they were determined upon and prepared for a civil war. ${ }^{26}$

Ce rapport, rédigé le 1er juillet, le lendemain des premiers incidents, démontre combien les événements s'étaient précipités et la situation s'était détériorée en moins de vingt-quatre heures. D'abord, la désobéissance collective du 30 juin ne s'était pas matérialisée subitement, sans antécédents. Quinze jours plus tôt, des habitants mécontents avaient prié le capitaine Thibodeau de leur dresser une requête afin de solliciter une exemption " $\mathrm{du}$ cinquième" - le tirage au sort d'un cinquième de la milice. L'inquiétude planait à ce sujet sur Pointe-Claire. Thibodeau se défila: il redoutait d'éveiller les soupçons du gouvernement à son

25 Témoignages de Pierre Denis et du milicien Jean-Baptiste Bonnet, et de Pierre Roi dit Lapensée, 19 août: 267-270.

26 Rapport du comité de Montréal, AC, PVCE, G: 254. 
égard et "de perdre sa place". ${ }^{27}$ L'affaire couva deux semaines, la tension se bandant jusqu'au point de rupture, à l'occasion de la chasse aux déserteurs. Et très tôt, le matin du 30 juin, des agitateurs avaient racolé des partisans avec diligence, afin d' "empêcher le Capitaine Leprohon" (sic), qui avait surpris deux déserteurs la veille. ${ }^{28}$

Enivrés par leur succès complet, ce jour-là, et aussi par leur propre audace, les chefs plus ou moins improvisés dilatèrent les cadres d'abord restreints de leur programme. Tant qu'à s'y mettre, pourquoi ne pas risquer le tout pour le tout? Ne pas libérer tous leurs miliciens en service à Laprairie, non plus les deux seuls réfractaires prisonniers! Pendant (et après) que certains pourchassaient Leprohon, d'autres fomentaient un véritable soulèvement: ils députèrent des messagers dans toutes les paroisses environnantes (jusqu'à Coteau-du-Lac) pour disséminer leurs mots d'ordre et convoquer les hommes à une manifestation monstre prévue pour le lendemain, à Lachine. Ils savaient éperonner les hésitants par des menaces appropriées.

Ainsi, Eustache Beneche dit La Victoire se dépensa sans compter pour la cause. Il pressait les habitants de l'accompagner en armes à Lachine, le lendemain, afin de franchir le fleuve à bord des bateaux du roi et de "chercher les miliciens incorporés" encore à Laprairie. ${ }^{29} \mathrm{Il}$ endoctrina d'autres émissaires. Ceux-ci proliféraient et sillonnaient à leur tour les paroisses avoisinantes en tous sens, même jusqu'aux Cèdres, "pour ... quérir des gens de bonne volonté". Le programme pour le lendemain ? Foncer sur Lachine en force, puis de là jusqu'à Laprairie afin de ramener les miliciens. Pourquoi ? Cent-cinquante habitants n'avaient pu pétitionner le gouvernement, par suite du refus de Thibodeau. Beneche convainquit Louis Brunet d'aller répandre la nouvelle à Lachine et d'y appeler les "gens de bonne volonté et se rendre à Côte des Neiges afin d'aller à Laprairie pour aller quérir ..." les miliciens. Comme on devait aussi présenter une requête,

27 Témoignages du capitaine Thibodeau, 20 août, et de François Rapin, 22 septembre: $278,306-307$.

28 Témoignage de Hyacinthe Proulx, 21 août: 283-284.

29 Témoignage de Pierre Valé, 20 août: 273. 
Brunet ne vit aucun mal à cela; non plus qu'à coopérer avec un Ducesneau, le lendemain, afin de grossir le nombre des partisans. Et à un Joseph Legault dit Deslauriers, tous deux expliquèrent que Leprohon avait lâché son prisonnier, que les autres miliciens ne se trouvaient pas à Laprairie sous l'autorité du roi, qu'enfin "ils étaient des gens perdus s'ils n'allaient pas à Laprairie les chercher ...". ${ }^{30}$

A mesure que la consigne et les rumeurs se véhiculaient de bouche en bouche, elles se dénaturaient, s'amplifiaient. Des griefs imaginaires prenaient corps dans l'esprit populaire, devenaient des certitudes propres à échauffer les passions. Les semeurs de désordre leur infusaient de la vraisemblance. La nuit tombée, le soir du 30 juin, on cabalait encore. On exhortait les pères à envoyer leurs fils, le lendemain: "si nous trouvons des jeunes gens par ici et les traverses sont payées, nous nous trouverons demain 800 hommes à Lachine . .." ${ }^{31}$

Dans beaucoup de cas, les témoins spécifient qu'on les a invités avec leurs armes. François Cousineau s'y refusa, ne voulant pas se conduire en "mauvais sujet". ${ }^{32}$ D'autres réagirent sans doute de la même façon: l'attroupement du ler juillet demeure bien en deça des possibilités, quant au nombre de ses participants. Mais certains furent enrôlés de force dans le mouvement: Hyacinthe Proulx, par exemple, le 30 juin, mena jusque chez Binet les déserteurs qui se terraient chez Jacques Trottier; Prégeau l'avait prévenu: "il fallait avoir les miliciens de Laprairie, puisqu'on n'avait pas le droit de les garder. [Et] ... s'il [Proulx] n'y allait pas, il n'en serait pas le mieux." Bazile Legault ne farda pas sa pensée d'un tel euphémisme: Si Proulx ne collaborait pas, "il [Legault] mettrait le feu à la maison et grange des père et mère du témoin". ${ }^{33}$

En général, on alléguait surtout quelques arguments passepartout: Leprohon n'avait pas le droit d'emprisonner les mili-

30 Témoignages de Jh Duchesneau, Louis Brunet et Joseph Legault dit Deslauriers, 20 août: 275-276, 277, 285.

31 Témoignage de J. Sauvé dit Laplante, 20 août: 277.

32 Témoignage de Fs Cousineau, 20 août: 277.

33 Témoignage de Hyacinthe Proulx, 21 août: 283-284. 
ciens tirés au sort; il fallait donc les reprendre; enfin, on composerait une pétition à ce sujet à l'adresse des officiers du gouvernement. D'autre part, le petit triomphe contre Leprohon enflait la vanité et l'outrecuidance des agitateurs, quelques-uns naturellement hâbleurs. Jean-Baptiste Sicard se vanta à Joseph Desautel que 500 hommes fondraient sur Laprairie, le lendemain. Desautel ricana que la simple apparition d'une compagnie de soldats suffirait à les faire détaler. “... ils n'avaient peur de rien, risposta Sicard, non désarçonné, et... ils étaient capables de manger tous les soldats qui leur seraient opposés." ${ }^{44}$

Tous ne militaient pas du même côté. Certains s'employaient à doucher cette ardeur égarée d'une partie de la population. Ainsi, le soir du 30 juin, Luc Courville ${ }^{35}$ aurait tenté de réfuter les fausses rumeurs en affirmant “qu'il était impossible d'avoir les miliciens parce que la loi était en force - il y avait plusieurs personnes présentes à cette conversation... [et] en parlant de l'affaire de Mr Leprohon, il a dit qu'il blâmait les habitants de ce qu'ils avaient fait, parce qu'il savait que la loi était en force qu'ils seraient punis comme Bte Julien et Lafrenière"; lui-même n'irait à Lachine le lendemain que pour remettre une requête aux autorités, "non pour user de violence". Le lendemain, en effet, le même homme conseillerait à un habitant de Vaudreuil de rentrer chez lui, vu “qu'ils n'auront jamais leurs gens commandés parce qu'ils l'étaient par la loi...": opinions impopulaires qui mécontentèrent plusieurs témoins de la conversation. ${ }^{36}$

Pierre Chamaillard convia même un Anglais, Robert McGregor, à se joindre à eux le lendemain. Ce dernier promit de le faire, si on lui prouvait l'illégalité de la levée de milice. Chamaillard lui divulgua que des "exprès" colportaient les nouvelles jusqu'aux paroisses de Vaudreuil, des Cèdres et du Grand Brûlé, afin que le lendemain, les habitants se déplacent au Coteau, et

34 Témoignage de Joseph Desautel, 22 septembre: 303.

35 Un Canadien marié et père de neuf enfants, en faveur de qui presque tous les témoignages concordent... sauf pour l'émeute du 1er juillet au soir. Ce qui ne lui épargnera pas la prison et de fortes amendes (voir plus loin).

36 Témoignages de Joseph Lalonde, Angélique Pilon, J.-Bte Gauthier et Eustache Beneche dit La Victoire, 22 septembre: 309-312. 
de là, jusqu'au faubourg Saint-Antoine et la porte de la ville. Puis, tous ensemble, "ils [demanderaient] quelques personnes qui leur diraient par quel droit les miliciens étaient retenus et si la loi était en force". McGregor et sa femme sondèrent davantage les intentions de Chamaillard. Leurs "représentants", confessa-t-il, étaient des Canadiens ; lui-même regagnerait son domicile, si la loi existait bel et bien, "et je crois que les autres en feraient autant". Selon McGregor, un témoin pondéré et objectif, Chamaillard, un "homme paisible et tranquille", semblait désireux de lui rapporter "comme une espèce de nouvelle - il paraissait bien curieux de savoir si la loi était en force et s'en était informé souvent... [D'ailleurs] plusieurs personnes paraissaient inquiètes et désiraient savoir la vérité... Chamillard lui dit qu'il avait entendu dire à des personnes bien instruites en ville qu'il n'y avait point telle loi." ${ }_{37}$ A l'exception des principaux perturbateurs, qui gouraient les autres, cette attitude semble avoir primé chez la majorité des habitants présents à l'échauffourée du lendemain.

Le premier juillet, durant toute la journée, des hommes de toutes les paroisses de la région (St-Charles, Pointe-Claire, SteGeneviève, Pointe-Bizarre, Ste-Anne, Vaudreuil, Les Cèdres, Coteau, etc.) marchèrent au rendez-vous. Suivant le mot d'ordre, bon nombre d'entre eux emportaient leurs armes: fusils, pistolets, bâtons. Presque tous les témoignages se recoupent curieusement quant aux buts recherchés par la majorité d'entre eux. Sur le chemin, ils "parlaient d'une requête à présenter au gouverneur au sujet des miliciens commandés". Plusieurs doutaient de la validité légale de la levée de milice. Leur nombre s'additionnait de ceux qu'ils rencontraient en route et qui consentaient à s'incorporer à cette masse mouvante. ${ }^{38}$

Mais il ne faut pas l'envisager comme une immense vague déferlant sur Lachine. Plusieurs attroupements s'étaient agrégés à divers endroits. Ainsi, vers 10 hres a.m., des hommes armés

37 Témoignage de Robert McGregor, 21 août: 285-287.

38 Témoignages de Jacques Sarazin, 20 août, de Jh Valé, 21 août: 281. Voir aussi divers autres témoignages en ce sens: 304, 305, 306, 307, 311-316, etc. 
trépignaient déjà à Lachine. Vers 11 heures, leur nombre s'était multiplié à plus d'une centaine. On s'encourageait mutuellement à aller enlever les miliciens à Laprairie. De temps à autre, des voix perçaient la rumeur diffuse de la foule: "s'il y en a quelquesuns qui s'en vont, nous mettrons le feu à leurs maisons." 39

McGregor, lui, tel qu'entendu la veille, parut chez Chamaillard. A la vue de plusieurs hommes armés, il leur décocha une question plutôt caustique: avaient-ils l'entention de "prendre" la ville d'assaut? A la suite de leur réponse - négative -, il leur conseilla d'envoyer plutôt cinq ou six personnes en ville afin d'éclaircir la question. "Allez-vous-en, lui jeta un Perrier, vous n'êtes pas des nôtres." McGregor enchaîna quand même "qu'ils devaient bien prendre garde à ce qu'ils allaient faire et de commettre aucune violence". Chamaillard voulut le rassurer: "Mr McGregor, ce n'est pas mon intention." D'autres réitérèrent les mêmes assurances. ${ }^{40}$

En vérité, Chamaillard avertit ses gens qu'il s'agissait de déterminer si le commandant du roi retenait illégalement les miliciens à Laprairie. A Pointe-Claire, Luc Courville s'évertuait lui aussi à arguer "que ceux qui voulaient présenter une requête devaient laisser leurs fusils et qu'ils ne devaient pas essayer de les avoir par force et qu'ils avaient été commandés de la part du roi"; "que ce n'était pas la manière de présenter une requête". Il faillit rentrer chez lui. Mais il déféra finalement aux pressions de ses amis et résolut d'attendre la réponse des autorités. Thivierge formula les mêmes réserves à propos des armes: "Mes enfants, il n'est pas nécessaire pour faire une requête de porter des fusils." ${ }^{41}$ Ces efforts ne réussirent pas à brider les forces qui commençaient à s'emballer.

Une partie du flot humain obliqua vers St-Laurent, afin d'en connaître davantage sur la loi de milice. Ils pausèrent chez le capitaine Binet: on voulait que "des gens respectables fussent

\footnotetext{
274, 284.

39 Témoignages de Pierre Valé et de Hyacinthe Proulx, 20 et 21 août:

40 Témoignage de Robert McGregor, 21 août: 286-287.

41 Témoignages de J.-L. Brunet, de J.-H. Pilon, Gilbert Leduc, Augustin Boileau et J.-Bte Massy, 22 septembre: 304, 312, 315-316.
} 
présents à une pareille assemblée". Selon certains témoins de Binet, ce dernier aurait désavoue le port des armes: il fallait les "laisser là", "on n'avait pas besoin de fusil pour une pareille requête", "c'est honteux de porter des fusils". ${ }^{22}$ Version improbable, puisque d'autres témoins distinguèrent Binet à Lachine, plus tard, le fusil à la main. ${ }^{43}$ Et la conduite du même capitaine de milice, la veille, ne cautionne pas en sa faveur.

Une fois à St-Laurent, ils persuadèrent Thibodeau d'aller consulter les hommes les plus respectables de Montréal afin de dissiper toute confusion autour de la loi de milice. Thibodeau ramassa par écrit leurs principales plaintes. D'abord, à leur avis, l'acte de milice n'était pas en opération. Deuxièmement, dans un tel cas, ils voulaient qu'on relâche les deux prisonniers de Montréal et les autres miliciens à Laprairie. Dans le cas contraire, ils livreraient immédiatement les déserteurs et sacrifieraient leur vie si nécessaire pour le gouverneur. Enfin, ils ne prisaient guère l'envoi, pour fouiller leurs maisons, d'hommes armés avec "ordre de tirer" sur "leurs gens" (i.e. probablement les déserteurs qui voudraient résister ou s'éclipser). Nantis de cette liste de doléances, Thibodeau et François Rapin se rendirent à Montréal. Ils devaient revenir vers 4 hres p.m. "aux 4 fourches du St-Laurent". En attendant, certains usèrent ce délai sur place. D'autres s'éloignèrent pour n'y retourner que plus tard. D'autres encore débattaient avec les habitants de St-Laurent l'opportunité d'élire deux habitants par paroisse pour énumérer leurs plaintes sur une liste, quitte à tout laisser tomber, si cela devait causer "du train”. D'autre part, là comme à Lachine, cette attente ne satisfaisait qu'une partie des participants. Un autre noyau voulait qu'on se concentre à Lachine avant même de pétitionner: stratégie naturelle chez des gens qui veulent étoffer leurs exigences de principe d'une politique de puissance. ${ }^{44}$ 316-318.

42 Témoignages de Jacques Trottier et J.-H. Meloche, 22 septembre:

43 Témoignages de A. Boyer et J.-L. Brunet, 22 septembre: 303-304.

44 Témoignages du capitaine Thibodeau, 20 août, Antoine Lange, François Rapin et Jacques Trottier, 22 sept.: 278-279, 296, 305-306, 317. 
Entre-temps, à Montréal, les membres du conseil exécutif analysaient les données du problème. Ils optèrent finalement pour la fermeté, afin de cautériser cette entorse aux lois, d'étouffer dans l'œuf cette fermentation, "this spirit of disobedience and outrage". Ils assignèrent deux agents "confidentiels" à la tâche d'argumenter avec "those mad people, to endeavour to bring them to a sense of their duty by delivering up the militia man that was rescued and further to insist that four of the most forward and who appear to be rig leaders in this revolt, to deliver themselves up to Justice, failing of which to forewarn them of the consequences and that an armed force will be sent to seize them". En cas d'insuccès, on ferait intervenir une centaine de réguliers, deux cents miliciens, ainsi qu'un détachement d'artillerie et deux canons. Les chefs devaient se plier à cet ultimatum et se rendre avant $6 \mathrm{hrs}$ p.m., le lendemain. Sinon, on embrayerait l'opération militaire. ${ }^{45}$

Ross et Louis Chaboillez, les deux agents du gouvernement, croisèrent peut-être Rapin et Thibodeau en route, sans connaître, évidemment, leur destination. Fait curieux, ceux-ci grignotèrent passablement de temps on ne sait trop où. On guettait leur retour pour 4 hres p.m. Mais lorsqu'ils se firent introduire par le colonel St-Dizier auprès de l'exécutif, chez le conseiller James McGill, il était près de 5 hres p.m. - s'il faut se fier à une note de McGill au bas de son rapport. Et pourtant, Thibodeau pensait encore à son retour pour 4 heures. Il égrena les motifs du mécontentement populaire, ajoutant que les habitants devaient se concentrer en un seul corps d'environ 1,500 hommes, à moins de deux lieues de la ville, et en détacher une dizaine de représentants pour parlementer avec le gouvernement. Cet homme, "a captain of militia”, écrit McGill, courroucé, se vit répartir par les conseillers qu'en tant que représentants du gouverneur, ils le chargeaient de transmettre leur réponse à ceux qui s'étaient massés aux quatre fourches, savoir: l'acte étant ratifié et en force, on devait l'exécuter; par conséquent, ils devaient se disperser immédiatement et céder les déserteurs; sinon, on les confronterait avec les

45 Rapport du comité de Montréal, AC, PVCE, G: 254-255. Etaient présents à cette réunion: James McGill, J. Monk et John Richardson. 
troupes du roi ; si toutefois ils désiraient d'autres renseignements, ils pouvaient déléguer trois ou quatre des leurs auprès du conseil. $^{46}$

$\mathrm{Au}$ retour, Thibodeau et Ross ne trouvèrent personne aux fourches. On les informa que les habitants s'étaient dirigés vers Lachine. Les deux hommes firent une halte chez un nommé Larose - peut-être pour manger. Ils ne reprirent la route qu'après le coucher du soleil. Et à Lachine aussi, ils allaient rater leur but: l'armée avait déjà nettoyé la place, ${ }^{47}$ redevenue paisible.

Pourtant, quelque temps auparavant, des coups de feu et des cris avaient lacéré cette paix. L'affaire avait démarré plus tôt. Voyant que Thibodeau ne reparaissait pas à l'heure prévue aux "quatre fourches", les plus timorés s'esquivèrent pour réintégrer leurs foyers respectifs. ${ }^{48}$ Vers le même moment, Ross et Chaboillez affrontaient à Lachine une cinquantaine d'habitants, dont plusieurs leaders de la résistance, soit Mallet, Bernard Courville, etc. Ils ne camouflaient pas leur objectif: "ravoir" leurs miliciens encore à Laprairie. Ils vitupéraient contre Leprohon, venu "jusque dans leurs greniers avec des tigeons de feu... accompagné de gens armés" à la recherche de fuyards. Les trois frères Courville maugréaient beaucoup, de même que Mallet. Ils firent alors mander par un cavalier le gros des forces, stationné à St-Laurent. ${ }^{49}$ Ce qui aiguilla la horde de protestataires vers la dernière scène de leur insoumission.

En peu de temps, les nouveaux arrivés inondent la place. Au courant de ce qu'on veut s'emparer des bateaux, le batelier de l'endroit, sur l'ordre de Grant, décalfète les trois qui restent. Sicard s'amène pour les faire mettre à l'eau. Le batelier l'informe que "ceux qui traversent dans les bateaux iront au fond, à quoi 281.

46 Ibid., 255-256, et témoignage du capitaine Thibodeau, 20 août: 279-

47 Témoignages du capitaine Thibodeau, 20 août, et de François Rapin, 22 septembre: $281,306$.

48 Voir témoignages: $315-316$ et passim.

49 Témoignages de Louis Chaboilliez et Amable Legault, 22 septembre: 296-297, 307. 
il [Sicard répond] ... qu'on les lui fera recalfater". ${ }^{50}$ La foule se gonfle et comme une meute affamée, se presse autour des deux représentants du gouvernement. Selon presque tous les témoins, elle se chiffre alors à plus de quatre cents hommes, dont près de deux cents armés de fusils, de pistolets et de bâtons. ${ }^{51}$

Ils s'attendent d'être 1,500 ou 2,000 le lendemain, signifientils à Ross et Chaboillez; ils veulent leurs miliciens et iront les ravir à Laprairie. L'un des modérés, Mallet, apaise momentanément le tumulte afin de permettre à Chaboillez de les haranguer: "Mes amis, écoutez les bons avis que ces Mess. veulent vous donner." A peine ce dernier ouvre-t-il la bouche qu'il se fait couper la parole par un extrémiste, Sicard. Mallet l'interrompt à son tour et s'interpose: "tais-toi, laisse les parler et donner leurs raisons et nous dirons les nôtres." Chaboillez les avise alors que la loi existe, qu'ils doivent s'y assujettir. Il se heurte à un mur, il ne peut les impressionner. Il leur enjoint de rompre les rangs, sinon ils se rendront coupables de rébellion. Mais eux accueillent ses propos par ce leitmotiv: "rendez-nous nos jeunes gens et nous nous en irons chez nous." - "C'est impossible", leur objecte Chaboillez. - "Eh bien, répondent-ils, nous forcerons pour les avoir"; le lendemain, à 1,500 ou 2,000, ils sauront bien imposer leurs conditions. ${ }^{52}$

Beaucoup se mirent alors à discuter d'une requête à rédiger. "N'écoutez pas ce que Mr Chaboillez va vous dire, leur cria Sicard, car il va vous gagner." Le tumulte s'amplifiait. Chaboillez décida de se retirer. Et alors qu'il amorçait un demi-tour avec sa voiture, "ils se mirent à crier Vive le Roi, nous sommes tous pour le Roi et nous marcherons tous ensemble, nos pères ne faisaient pas l'exercice et nous ne voulons pas d'exercice". Chaboillez, scandalisé, dut bondir: il leur lança qu'en l'occur-

50 Témoignage de J.-H. Fluet, 22 septembre: 305.

51 Voir témoignages: 275, 278, 289, 297, 299, 301, etc. Deux témoins seulement parlent de 200 hommes (voir témoignages: 285, 295). Le Montreal Herald du 4 juillet hazarde le chiffre de 400 , dont 80 armés. John Grant, lui, mentionne que "le tiers" portaient des armes (témoignages de John Grant, 20 août: 278).

52 Témoignages de J.-D. Lacroix, A. Lange et Louis Chaboillez, 22 septembre: $289-290,293,296-298$. 
rence, leur exclamation ("vive le roi") équivalait à "un blasphème". ${ }^{53}$ Apparemment, cependant, il se serait mépris sur leur intention. Selon un autre magistrat, J.-D. Lacroix, Mallet aurait déclaré, après les semonces de Ross et Chaboillez: "je vous remercie des bons conseils que vous nous donnez." Un certain nombre se dégagèrent alors du corps principal en criant: "Vive le Roi !" "Chaboillez, raconte le témoin, s'est retourné en leur disant que c'était une insulte. Mais le témoin a cru que c'était ignorance de leur part." ${ }_{54}$ Et Chaboillez lui-même s'amende implicitement plus loin, après avoir résumé les principales causes du malaise populaire et caractérisé la conduite générale des gens, avant l'intervention de la troupe:

Un des Courville paraissait fort animé et disait qu'on méprisait les Canadiens et qu'il était temps de montrer ce qu'ils étaient - ... Luc Courville [son frère] ... ne paraissait pas approuver tant de violence et disait que cela finirait mal, que ce rassemblement était suffisant pour causer de l'effroi et faire craindre les conséquences qui pouvaient en résulter. Il a paru au témoin qu'il y avait un mécontentement général parmi les gens ainsi attroupés ils se plaignaient qu'on leur fit faire l'exercice et qu'ils étaient mal nourris. Ils se sont comportés d'ailleurs très polis et n'ont commis aucune injure. Ils demandaient seulement leurs prisonniers. Mallet, dans sa conversation avec le témoin, a paru très modéré. Il paraissait seulement fort en colère contre le Maj. Leprohon qui était venu chez lui armé avec son propre frère pour poursuivre des prisonniers. ${ }^{55}$

Ce témoignage est confirmé par ceux de plusieurs magistrats de Montréal, venus prêter main forte à Ross et Chaboillez. Lacroix, Ogilvie, Gordon, Grant, McCord et divers curieux, v.g. Ant. Lange, tentèrent de désamorcer cette masse explosive, de raisonner les habitants et les renvoyer chez eux. Ils les sermonnaient sur leur conduite criminelle, sur la synchronisation on ne peut plus mauvaise dans le choix du moment pour adresser des

53 Témoignage de Louis Chaboilliez, 22 septembre: 297.

54 Témoignage de J.-D. Lacroix, 22 septembre: 292.

55 Témoignage de Louis Chaboilliez, 22 septembre: 298-299. 
requêtes, etc. Les habitants ne bougeaient pas, rivés sur place par leur volonté commune d'attendre Thibodeau et de préparer cette fameuse requête. D'autres ne s'embêtaient pas de nuances ni d'excuses. Ils voulaient leurs miliciens: ou on les leur produirait, avec ou sans pétition, ou ils iraient les rafler de force "morts ou vifs". La majorité conjecturait ou feignait de croire que la loi n'existait ou ne s'appliquait pas. Et si leurs représentants les avaient "vendus", ce n'était pas une raison de lâcher leurs jeunes gens. Quant à Thibodeau, s'il échouait dans sa mission, “ils étaient déterminés d'aller à Laprairie retirer leurs gens, ce qui paraissait l'opinion générale ..." 56

Quelques modérés cherchèrent encore à endiguer les forces déjà hors de contrôle, prêtes à se déchaîner. Sur l'assurance de Lacroix qu'il consignerait leurs idées dans une pétition, dès le lendemain, Vallé et quelques autres partirent se désarmer. "Il a paru ... qu'un nombre s'est alors dispersé et qu'il ne restait pas plus de 200 à 250 personnes." 57 Mallet aurait détourné 200 hommes avant le coucher du soleil: chiffre un peu exagéré. Et après une conversation avec Ogilvie, il lui aurait serré la main en disant à son entourage: "allons-nous en, mes enfants." Luc Courville et vraisemblablement d'autres comme lui faisaient jouer leur influence. Même Binet, après le discours d'Ogilvie, aurait changé son fusil d'épaule: “mes enfants, on n'a point à répliquer sur les bonnes raisons de ces messieurs, je crois qu'il est à propos de nous retirer..." Et "ils se sont retirés ... plusieurs". ${ }^{58}$

Fait curieux, qui intrigue tout de même l'historien, tous ces "bons" citoyens semblaient très actifs dans la bande d'hommes qui occupait les lieux, peu avant le combat. D'après McCord, Mallet "paraissait très animé et changeait souvent de place... il paraissait que la foule l'écoutait avec attention". ${ }^{59}$ Pour sa

56 Témoignages de J.-D. Lacroix et E. Lange, 22 septembre: 292, 293294 ; aussi plusieurs autres témoignages, 20 août et 22 septembre: 278,293 , 302 , etc.

57 Témoignage de J.-D. Lacroix, 22 septembre: 291.

58 Témoignages de J.-L. Brunet, Jh Pilon, Jh Letang, Louis Triolet et $\mathrm{Jh}$ Théorêt, 22 septembre: 304, 312, 318-319.

59 Témoignage de John McCord, 22 septembre: 302. 
part, Luc Courville - comme d'autres d'ailleurs — avoua à Lacroix qu'il ne pouvait tourner les talons "parce qu'ils étaient guettés et qu'il y avait une garde pour les empêcher de retourner". Et pourtant, un autre témoin identifie nommément Luc Courville et Mallet comme ceux qui auraient posté des sentinelles "dans le chemin pour empêcher le monde de se retirer". Et lorsque ce témoin voulut le faire, ils lui auraient couru après en hurlant: "retournez, avancez, ils ne vous feront pas de mal, parlant des troupes ..." Courville et d'autres "ont dit qu'il fallait mettre une garde sur le chemin pour empêcher le monde de s'en aller pour attendre Mr. Thibodeau et voir comment ça se passerait après sa réponse". ${ }^{60}$ Cette dernière phrase dénoue peut-être la contradiction apparente des témoignages. Car autrement, comment déchiffrer l'énigme, au moins dans les cas de Mallet et de Luc Courville. A moins que Boyer ne se parjure: il prétend n'avoir pas aperçu Sicard, 'Thivierge et le Capitaine Binet sur les lieux ! Peut-être aussi confond-il les trois Courville. En tout cas, Mallet admit à Ante Lange qu'il ne pouvait refréner la foule, trop déterminée à persister dans sa conduite. Et Luc Courville "paraissait mortifié de se trouver dans l'assemblée... il dit aussi que s'il s'en allait, il y avait une garde qui l'arrêterait et qu'il courait le risque que sa maison soit brûlée". ${ }^{61}$

John Grant convainquit plus de quatre-vingts habitants de déposer leurs armes le long de la clôture. Mais "quelques-uns les ont rappelés et ils ont repris leurs armes". La plupart des manifestants lui certifiaient qu'ils ne désiraient infliger de mal à personne. Mais ils voulaient ravoir leurs jeunes gens et faire savoir leur point de vue au gouvernement. ${ }^{62}$

Donc, en fait, des mobiles différents, se reverbérant dans des attitudes dissemblables, tiraillaient intérieurement cette cohue désordonnée. Peut-être se serait-elle désintégrée d'ellemême avec un peu plus de temps. En tout cas, les départs et cette 295-296.

60 Témoignages de J.-D. Lacroix et de Ante Boyer, 22 septembre; 298,

61 Témoignage de Ante Lange, 22 septembre: 295. $278,299$.

62 ler et 2ième témoignages de John Grant, 20 août et 22 septembre: 
absence de cohésion tranquillisèrent les appréhensions du magistrat Lacroix. Estimant le danger passé, il s'apprêta à retourner à Montréal. C'est à ce moment que Vallé et Luc Courville, peutêtre à la demande des habitants, vinrent le trouver pour se renseigner sur l'heure pour laquelle il les convoquait, le lendemain, à propos de la pétition. Thomas Bleau, un autre notable, venait tout juste de les engager à partir, et Courville, de reconnaître: "nous ferons mieux de nous retirer." Lacroix ordonna alors leur arrestation. "Vous n'aurez pas de peine à me prendre, commenta Courville, parce que je m'en allais et que je ne voulais pas rester avec les autres." Lacroix les relâcha "sur la promesse qu'ils viendraient à Montréal". Durant sa courte captivité, Courville persévéra dans sa version, cette fois auprès de James Fraser: il n'avait "joint les autres ... [que pour] présenter une requête". Fraser l'avait d'ailleurs repéré auparavant dans la foule. Courville lui avait alors semblé plus paisible que les autres. ${ }^{63}$

C'est à ce moment critique, ce point tournant, qu'intervint le magistrat John McCord. Il précédait les troupes dépêchées par le conseil exécutif. Il se mit à chapitrer les habitants: ils s'abandonnaient à la démence; leur rassemblement était illégal et dangereux; s'ils voulaient pétitionner, ils devaient s'y prendre autrement et lui déléguer cinq ou six représentants le lendemain et lui-même s'en occuperait, même s'il doutait des résultats. Ils "ont répliqué qu'ils voulaient avoir leurs gens et qu'avec requête ou sans requête, il les aurait toujours" ". ${ }^{4}$ Ils rabâchèrent leurs mêmes excuses pour avaliser leurs actes: inexistence de la loi, ni ratifiée ni promulguée devant les miliciens, donc imposée de façon inconvenante, anormale; ils se sacrifieraient volontiers, cependant, si le roi avait besoin d'eux pour la défense du pays.

McCord lut alors la loi contre les attroupements, puis, au nom du roi, les somma de se disperser. ${ }^{65}$ Certains obtempérèrent

63 Témoignages de J.-D. Lacroix, Thomas Bleau, J. G. Degen et James Fraser, 22 septembre: 290-291, 313, 311. 301.

64 Témoignages de Ante Lange et John McCord, 22 septembre: 293,

65 Montreal Herald, 4 juillet 1812. 
à cet ordre formel. Mais d'autres se gendarmèrent davantage, menaçant de prendre les bateaux à Lachine. "Non certainement, les défia McCord, vous ne les [les jeunes gens] aurez pas, voilà les troupes qui viennent pour vous [en] empêcher." Et en effet, les soldats, surgis sur la route, se rangèrent devant la foule tumultueuse pour la rembarrer. McCord suspendit leur entrée en action durant un délai d'une demi-heure. Entre-temps, ses efforts de dissuasion n'aboutirent pas. ${ }^{66}$

Cela n'avait rien de surprenant. L'ultimatum cravachait leur entêtement et scellait le sort - jusque-là peut-être incertain - des événements : on pressait la gachette, on embrasait l'huile. Les habitants allaient-ils se déjeter ainsi devant les troupes, répudier d'un coup leur attitude et se débiner la tête basse ? Et les chefs, ne pouvant plus pactiser avec les forces de l'ordre, se cuirassèrent dans leur attitude. Au procès, le Capitaine Ducharme soutint que "les habitants paraissaient avant cette affaire inquiets sur la loi de la milice et ne savaient pas si la loi était en force". Il qualifia "d'irréprochable" la conduite de Mallet et de la plupart des autres, sauf qu'ils "se soutenaient dans une mauvaise cause", ${ }^{67}$

Quoi qu'il en soit, on invectivait apparemment ceux dont le courage ou la conviction croulait: "lâches, laissez au moins vos fusils pour que nous venions à bout de ce que nous avons commencé." Sicard, passablement éméché, associait absurdement ennemis américains et soldats britanniques: "si elle [la guerre avec les États-Unis] n'est pas déclarée, nous allons la déclarer"; quant aux troupes, "nous ne [les] craignons pas ... et nous allons les repousser".68 Apparemment, sa cervelle divaguait, embrumée par l'alcool ou la fièvre du moment. Peut-être qu'au fond sourdait la rancœur que cuvaient difficilement les Canadiens depuis plusieurs générations contre les "Bostonnais", fourrant dans le même sac Américains et Britanniques, ces anciens alliés 301.

66 Témoignages de Ante Lange et John McCord, 22 septembre: 293,

67 Témoignage du Capt. Ducharme, 22 septembre: 300-301.

68 Témoignages du Capt. Ducharme et de Ante Lange, 22 septembre: 300-301, 294. 
naturels, ces conquérants et fossoyeurs de la Nouvelle-France. Ou enfin Sicard et plusieurs autres bisquaient tout simplement par suite de la conscription; et dans leur colère et leur mauvaise conscience, ils se débondaient à propos de tout et de rien.

McCord renouvela vainement ses injonctions à deux autres reprises, soit après quinze, puis 25 minutes. Des Canadiens fléchirent et déguerpirent par la route; ${ }^{69}$ mais la plupart demeurèrent déployés, face aux soldats, soit par conviction, par obstination ou rodomontade. Certains s'engageaient à regagner leurs foyers, mais seulement "si les troupes se retiraient" aussi. Les trente minutes de grâce expirées, les soldats rompirent la tension par un coup de canon au-dessus de la tête des habitants, en direction du fleuve. Ceux-ci, jusqu'alors "très tranquilles", n'ayant houspillé personne ni commis "aucune violence personnelle", ripostèrent avec une quinzaine de décharges en direction des soldats. $^{70}$ Le crépitement sporadique des coups de feu jaillissant des deux côtés - environ soixante en tout -, ne dura que quelques minutes. Avec un mort et un blessé grave dans leurs rangs, les Canadiens profitèrent de l'obscurité pour détaler dans toutes les directions à travers les bois. ${ }^{71}$ Peu après, Thibodeau et Rapin survinrent sur les lieux. Le fracas de l'escarmouche s'était évanoui. Seuls le silence et Mallet, encore là, les accueillirent. Ce dernier leur narra les faits marquants de la journée et expliqua sa présence par la nécessité de transporter et de soigner le blessé. $^{72}$

69 Voir témoignages: passim.

70 Témoignage de J.-D. Lacroix, 23 septembre: 291. Ce dernier semble ainsi désapprouver implicitement le recours aussi rapide à la force militaire. Un autre témoin, John Grant, résume laconiquement l'accrochage des deux forces en présence: "les personnes ainsi assemblées ont tiré sur les troupes et n'ont été dissipées que par force." Toutefois, il précise presque aussitôt qu'il ignore d'où provenaient les premiers coups de feu (Témoignage de John Grant, 20 août: 278).

71 Témoignage de John McCord, 22 septembre: 302. Prévost à Liverpool (no 56), Montréal, 6 juillet 1812, AC, Q.118:36. Le Montreal Herald, du 4 juillet 1812 félicite les troupes et leurs officiers pour leur sang-froid et leur soin à épargner les vies humaines - témoignage que leur auraient d'ailleurs rendu des Canadiens en pleurs, peu après la fusillade ( $i b i d$. .).

72 Témoignage de François Rapin, 22 septembre: 306. 
Le lendemain matin, 2 juillet, des soldats réguliers, épaulés de détachements de la milice de Montréal, soit environ 450 hommes "who turned out with an alacrity highly creditable to them", entreprirent de râtisser en vitesse les différentes paroisses de la région. Ils pêchèrent ainsi vingt-quatre des principaux insurgés et les incarcérèrent à Montréal. ${ }^{73}$ Cette marche forcée sur l'île de Montréal (particulièrement fructueuse à PointeClaire et à St-Laurent) avait duré trente heures. Les troupes avaient parcouru quarante-cinq milles, dont les vingt-deux derniers sans repos ni nourriture. Les 24 prisonniers s'additionnaient à la dizaine d'autres cueillis le soir de l'émeute et à ceux qui se trouvaient déjà à Montréal, soit un total de trente-sept. ${ }^{74}$

Le 4 juillet, les miliciens penauds vinrent se soumettre publiquement et faire amende honorable: environ trois cents "of these misguided men voluntarily came in to make their submission to me ${ }^{75}$ acknowledging their guilt and faithfully promising immediately to comply with the order for their being drafted and giving me the strongest assurances of their readiness in future to perform whatever service I might require of them". ${ }^{76}$ "His Excellency expostulated them as a father and pointed out to them the danger of their situation, in a style truly honorable to his feelings, assuring them his forgiveness on delivering up those who had been the promoters of the insurrection... and the militia deserters..." 77 Parmi cette troupe - environ deux cents personnes selon Brenton - les magistrats ne purent pointer du doigt que deux individus "as having taken an active part

73 Prévost à Liverpool (no 56), Montréal, 6 juillet 1812, AC, Q.118: 37.

74 Ibid.; Montreal Herald, 4 juillet 1812.

75 Prévost avait quitté Québec le 1er juillet et venait d'arriver à Montréal, afin d'être à proximité des opérations militaires éventuelles (voir la lettre de Prévost à Liverpool (no 56) déjà citée, 35, et La Gazette de Québec, 2 juillet 1812).

76 Prévost à Liverpool (no 56), ibid., 37. Le gouverneur ajoute que l'on interroge les prisonniers et qu'on traduira les plus coupables devant les tribunaux. Il fait erreur toutefois quant à la date de l'acte public de soumission: dans cette lettre, datée du 6 juillet, il dit 'yesterday" (ibid.). Pourtant, la lettre de son secrétaire militaire Brenton au conseil exécutif de Québec, datée de Montréal, 4 juillet, raconte cette cérémonie (voir AC, PVCE, G: 273).

77 Montreal Herald, 4 juillet 1812. 
in the late dissafection. They have been committed to prison; the remainder returned home apparently deeply impressed with a sens of their situation and His Excellency's lenity towards them and solemnly engaging to send to Laprairie the men who had left their quarters and to be forthcoming themselves for any service to which His Excellency might think proper to appoint them." 78

$$
\text { (à suivre) }
$$

JEAN-PIERRE WALLOT, Département d'histoire, Université de Montréal.

${ }^{78}$ Brenton au conseil exécutif de Québec, AC, PVCE, G: 273-274.

A la Commission des lieux et monuments historiques du Canada. - L'un de nos membres-correspondants, M. JeanJacques Lefebvre, archiviste en chef à la Cour supérieure de Montréal, a été nommé récemment membre de la Commission des lieux et monuments historiques du Canada. M. Lefebvre est l'un des deux représentants du Québec au sein de cette Commission. Félicitations et vœux de succès. 